\title{
Propuesta de indicadores para evaluar la sostenibilidad de la actividad turística. El caso del Valle de Viñales (Cuba)
}

\author{
Yolanda PÉREZ AlBERT \\ Departamento de Geografía, Grupo de Investigación GRATET \\ Universidad Rovira i Virgili \\ myolanda.perez@urv.cat \\ Marta NEL·LO ENDREU \\ Departamento de Geografía, Grupo de Investigación GRATET \\ Universidad Rovira i Virgili \\ martagemma.nello@urv.cat
}

Recibido: 3 de octubre de 2012

Enviado a evaluar: 22 de enero de 2013

Aceptado: 22 de marzo de 2013

\section{RESUMEN}

El Valle de Viñales (Pinar del Río) se encuentra situado en el sector occidental de Cuba y es una de las opciones más valoradas como destino turístico de naturaleza de la isla. Su interés queda reconocido a partir de diferentes figuras de protección como Paisaje Cultural de la Humanidad (1999, Comité de Patrimonio Mundial de la UNESCO) o Parque Nacional de Viñales (2001, Sistema Nacional de Áreas Protegidas). Esta zona rural convive con un modelo de desarrollo turístico incipiente y a pequeña escala, que tiene como finalidad el incremento del nivel de vida de la población local así como en la preservación de sus recursos naturales y culturales. El objetivo de este trabajo es valorar la sostenibilidad de la actividad turística implantada en Viñales mediante la aplicación de un sistema de indicadores que permitan establecer una serie de recomendaciones que mejoren la sostenibilidad de la actividad turística.

Palabras clave: Ecoturismo, indicadores, desarrollo sostenible, Valle de Viñales, Cuba.

\section{Proposed indicators for assessing the sustainability of tourism. The case of Viñales Valley (Cuba)}

\begin{abstract}
Viñales Valley (Pinar del Rio) is located in the west of Cuba and is one of the most valued natural tourist destinations of the island. Its interest is recognized based on different categories of protection as a Cultural Landscape (1999, World Heritage Committee, UNESCO) or Viñales National Park (2001, National System of Protected Areas). This rural area coexists with a small-scale emerging model of tourist development, which aims to increase the standard of living of the local population and the preservation of its natural and cultural resources. The objective of this study was to assess the sustainability of tourism in Viñales applying a system of indicators to establish a series of recommendations to improve the said sustainability of tourism.
\end{abstract}

Key words:Ecotourism, indicators, sustainable development, Viñales Valley, Cuba 
Les indicateurs proposés pour l'évaluation de la durabilité du tourisme. Le cas vallée de Viñales (Cuba)

\section{RÉSUMÉ}

La Vallée de Viñales (Pinar del Rio) est située a l'ouest de Cuba et est une des destinations touristiques de nature les plus prisées de l'île. Son intérêt est reconnu par différentes formes de protection comme le Paysage Culturel (1999, Comité du Patrimoine Mondial, UNESCO) ou le Parc National de Viñales (2001, Système National des Aires Protégées). Cette zone rurale coexiste avec un modèle du développement du tourisme, émergent et à petite échelle, qui vise à augmenter le niveau de vie de la population locale et la préservation de ses ressources naturelles et culturelles. L'objectif de cette étude est d'évaluer la durabilité du tourisme à Viñales en appliquant un système d'indicateurs pour établir une série de recommandations qui visent à améliorer la durabilité du tourisme.

Mots clés: Ecotourisme, indicateurs, développement durable, Vallée de Viñales, Cuba.

\section{INTRODUCCIÓN}

Que el turismo contribuye al crecimiento y a la diversificación económica es algo reconocido por todos aquellos que trabajan en el sector pero, con menos frecuencia, se asume que éste también genera una serie de impactos adversos tanto en la esfera medioambiental como social y cultural. El turismo, sobre todo en los países en vías de desarrollo, se aprecia como una potente herramienta con capacidad para generar puestos de trabajo, favorecer la creación de empresas locales, incrementar el ingreso de divisas, fomentar la inversión privada, etc., aspectos positivos que se imponen sobre los problemas que esta actividad pudiera generar (Nel-lo y Pérez, 2007). Para salvaguardar la sostenibilidad medioambiental y social es necesario que cualquier región que pretenda iniciar o implantar una actividad turística realice una planificación adecuada con el objetivo de, por un lado, maximizar sus beneficios y, por otro, minimizar sus impactos negativos (Priskin, 2001). Además del objetivo original, la protección del entorno natural y social, la sostenibilidad turística también es vista por muchos gobiernos como una medida que permite definir un nuevo modelo turístico, que tiene como objetivo final el incremento de la competitividad del destino (Blancas et al., 2009). Para conseguir la viabilidad del turismo en un territorio dado es necesario promoverlo de manera sostenible, ligado a la calidad de vida de sus habitantes, a la protección del medio ambiente y del patrimonio cultural (Vázquez y Martín, 2001).

Según Saarinen (2006) se pueden identificar tres aproximaciones a la idea de turismo sostenible a escala local. La primera de ellas trata de identificar los cambios negativos producidos por el turismo en el capital natural y cultural del destino. Este primer enfoque tiene como eje central los recursos (naturales, sociales, culturales) y su conservación, estableciendo límites al crecimiento del turismo. La segunda se basa en la actividad y manifiesta que el turismo es un sector que necesita y depende de los recursos, tanto en el presente como en el futuro, de modo que su conservación es clave para preservar el capital económico invertido en él. Su objetivo es mantener el turismo y los recursos en los que éste se basa para garantizar las necesidades futuras de la industria. La tercera aproximación, sustentada en el capital social local, postula 
por una mayor participación de los diferentes actores, especialmente las comunidades de acogida, de modo que se fomente su desarrollo de forma integral.

Los tres enfoques anteriores, establecen una dependencia clara entre turismo y recursos naturales y culturales. Esta dependencia queda reflejada en "La Teoría de la Autodestrucción del Turismo" (Holder, 1988; Weise, 2000; Barbosa, 2003) que sostiene que el turismo, implantándose bajo criterios no sostenibles, se desarrolla y declina en cuatro etapas: (1) En la primera, población con ingresos elevados se instala en una localización remota con valores naturales privilegiados, viviendo completamente aislada de la población local. (2) En la segunda, la promoción atrae a turistas de ingresos medios de modo que, ante la presión de la demanda, se incrementa la oferta de equipamientos y servicios y la población local trabaja en el sector turístico abandonando la producción agrícola tradicional. El incremento de la oferta conlleva una reducción de los precios de modo que se produce un deterioro del producto emigrando los turistas más adinerados. Se llega a la (3) tercera etapa cuando se instala un turismo de masas dirigido a un sector de la población con ingresos menores y procedentes de estratos sociales inferiores. En la (4) cuarta etapa surgen conflictos medioambientales (producción de residuos, generación de contaminantes, destrucción del entorno natural...) y sociales (desempleo, infraempleo, deterioro de la cultura local...) de modo que la afluencia de turistas es nula. Esto supone que se abandonan las infraestructuras turísticas, el entorno medioambiental permanece afectado negativamente sin ingresos para poder recuperarlos y la población local es incapaz de retomar el estilo de vida tradicional (Figura 1).

Sea cual sea la aproximación a la idea de sostenibilidad en el turismo o la visión más o menos catastrófica de su evolución, varios son los autores que mantienen que esa sostenibilidad de la actividad turística es más un aspiración o deseo que un objetivo alcanzable y mesurable (Middelton y Hawkins, 1998, citados por Ko, 2005). Además, es manifiesto que las propuestas institucionales y académicas que tienen como objetivo la sostenibilidad del turismo son muy numerosas pero los avances son muy lentos y los resultados escasos (Comisión para la sostenibilidad del turismo europeo, CCE, 2003 citado por Vera e Ibars, 2004).

Uno de los objetivos planteados desde el ámbito político-administrativo y académico, es el diseño de metodologías y la selección de indicadores que permitan medir el grado de sostenibilidad de la actividad turística. En este sentido, el turismo se considera una forma de desarrollo y por lo tanto, para evaluarlo se pueden aplicar los mismos criterios que se aplican al desarrollo en general. En estos términos, la sostenibilidad del turismo puede ser entendida como la capacidad que detenta un sistema para mantener el estado de salud necesario para sobrevivir, obteniendo progresivamente mayores niveles calidad en todos los ámbitos. En este caso, el "estado de la salud" se define como la situación deseable y aceptable de un sistema de modo que le permita su supervivencia (Ko, 2005). 
Figura 1. Teoría de la autodestrucción del turismo.

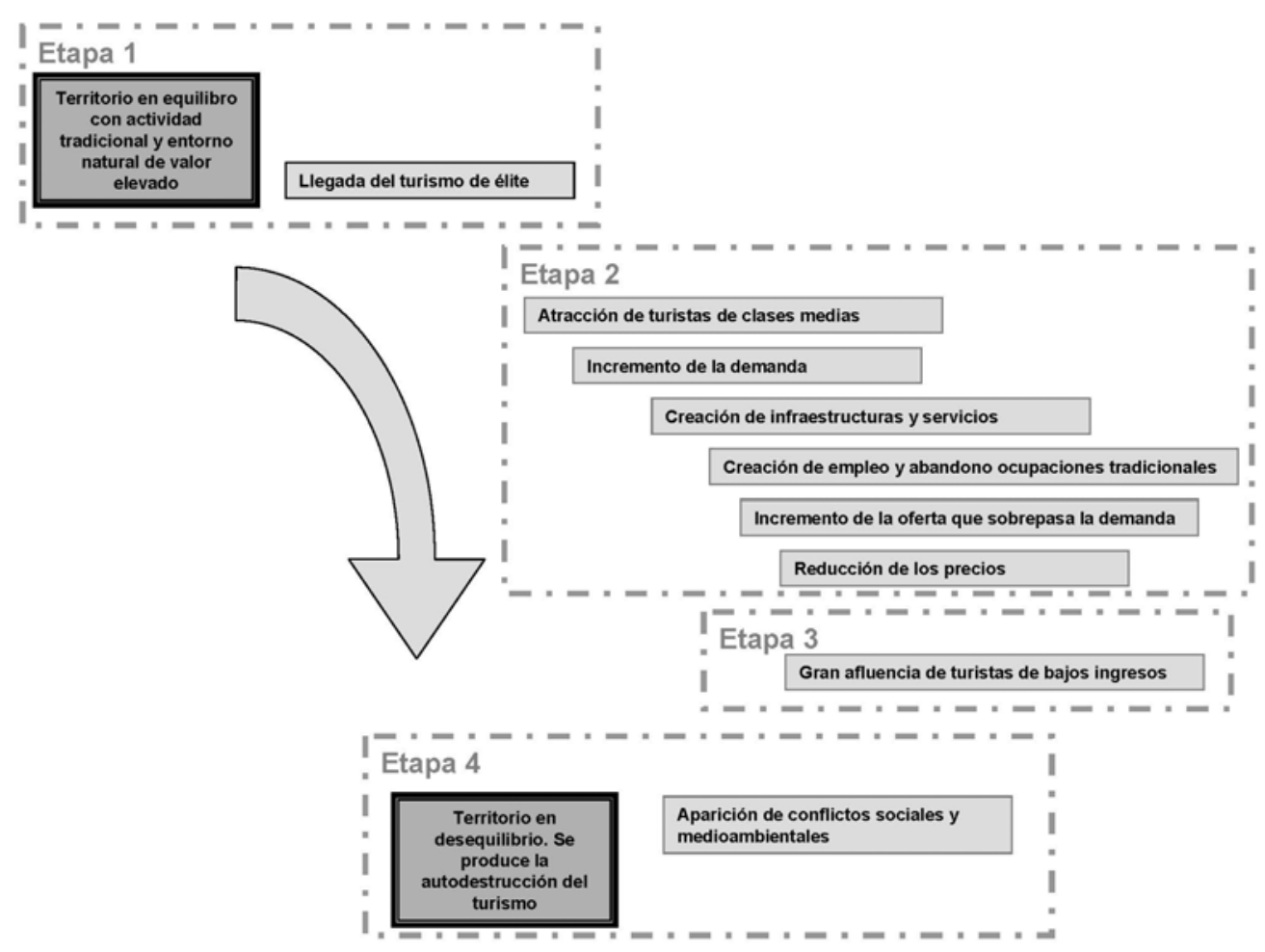

Fuente: Elaboración propia.

Existen diferentes trabajos que tienen como objetivo definir y aplicar una serie de indicadores que permitan medir el grado de sostenibilidad de la actividad turística en un destino. Nos podemos remontar a los primeros indicadores claves y suplementarios según el tipo de destinos turísticos que propuso la OMT (1998) y que han ido ampliándose y complementándose posteriormente en OMT (2005). Más recientemente se puede hacer referencia a los estudios de Pérez et al. (2009) que analizan la sostenibilidad del turismo rural en 13 zonas turísticas rurales de Andalucía y que abarcan 201 municipios; la contribución de Blancas et al. (2009) en la que propone un sistema de indicadores sintéticos a nivel local que proporcionen una medición multidimensional de la sostenibilidad turística y que es aplicado a los municipios de Andalucía con relevancia como destino turístico cultural. El trabajo metodológico de Choi y Shirakaya (2006) trata de obtener, a partir de la colaboración de un grupo extenso de expertos, un sistema de indicadores para evaluar el desarrollo derivado del turismo comunitario. Algunos de ellos tienen en cuenta el grado de madurez del destino como 
son los trabajos de Vera e Ibars (2004) con la definición de un conjunto de indicadores de sostenibilidad de destinos maduros aplicados a determinados municipios de la comarca del Bajo Segura en la provincia de Alicante (España) o la contribución de Sancho (2007) que realiza una comparativa de indicadores de sostenibilidad en destinos turísticos desarrollados, en desarrollo y en poblaciones con riesgo de exclusión.

En la región del Caribe y, concretamente en el caso de Cuba, se ha avanzado durante la última década en la definición de un sistema de indicadores adaptado al entorno para medir la sostenibilidad del turismo. Se destacan la propuesta de la Asociación de Estados del Caribe (Díaz y Norman, 2004), la de organismos cubanos como la del Ministerio de Turismo y el Ministerio de Ciencia Tecnología y Medio Ambiente (2003) así como estudios recientes donde se evalúa la sustentabilidad del turismo en diferentes destinos de Cuba (Salinas et alt., 2008; Caballero et al., 2010).

\section{2. ÁREA DE ESTUDIO Y OBJETIVOS}

El municipio de Viñales situado en la zona norte-central de Pinar del Río, la provincia más occidental de Cuba, limita al norte con el Golfo de México; al este con el municipio La Palma, en su extremo más meridional, con los municipios Consolación del Sur y Pinar del Río, y al oeste con el municipio de Minas de Matahambre. Tiene una extensión de $704 \mathrm{~km}^{2}$, de ellos, $697 \mathrm{~km}^{2}$ son de tierra firme y los $7,9 \mathrm{~km}^{2}$ restantes son de cayos litorales, lo que representa el $6 \%$ del área total de la provincia. Se encuentra a $25 \mathrm{~km}$ de la capital provincial, Pinar del Río, y a $178 \mathrm{~km}$ al oeste de la ciudad de La Habana.

En el municipio predomina el relieve montañoso, que alcanza el $71 \%$ de la superficie total, caracterizado por una serie de elevaciones cársticas, del periodo jurásico inferior o medio, conocidas como mogotes. Éstos son formaciones únicas en la isla, que pueden llegar a alcanzar centenares de metros de altura (Melero, 2005). En el Valle de Viñales existen otras elevaciones constituidas por las alturas de pizarras, las más antiguas de las existentes en el país y también del área caribeña. Relacionados con estas formaciones se encuentran los sistemas cavernarios más extensos de Cuba como Palmarito, el mayor complejo de cavernas hasta el momento estudiado en Cuba, Sierra Guasasa y la Gran Caverna de Santo Tomás (Hernández y Ramírez, 2005). En el interior de la mayoría de estas cuevas se ha hallado un elevado número de restos fósiles, evidencias arqueológicas y paleontológicas de más de dos millones de años. A estos valores geológicos y paleontológicos se suman los extraordinarios recursos de una fauna y una flora muy ricas marcadas por un fuerte endemismo (Rigol, 2005).

El municipio de Viñales cuenta con una población total de 27.806 hab. (2009) distribuida en 33 asentamientos y con una de densidad $33,5 \mathrm{~h} / \mathrm{km}^{2}$. La base económica del municipio es la actividad agropecuaria y el cultivo del tabaco, aunque también destaca la actividad forestal y, en estos últimos años, la turística.

Por la conjunción de sus valores naturales y culturales, el Valle de Viñales fue declarado en el año 1999 lugar Patrimonio de la Humanidad en la categoría de Paisaje Cultural. En el municipio también se encuentran espacios decretados monumentos 
nacionales, distinción otorgada a la Villa de Viñales, a la Gran Caverna de Santo Tomás y a la Palma Corcho entre otros. Más recientemente, en 2001, se creó el Parque Nacional Viñales, administrativamente localizado en el municipio de Viñales, con una pequeña porción de la zona de amortiguamiento en el municipio de Minas de Matahambre.

El objetivo de este trabajo es, en primer lugar, realizar la selección de un sistema de indicadores adaptados a un destino turístico incipiente que permita valorar su sostenibilidad respecto al ámbito medioambiental y social. En segundo lugar, realizar la aplicación práctica del conjunto de indicadores al destino turístico del Valle de Viñales (Cuba) con la intención de evaluar su sostenibilidad actual y realizar propuestas de mejora ${ }^{1}$.

\section{METODOLOGÍA: SISTEMA DE INDICADORES PARA EVALUAR LA SOSTENIBILIDAD TURÍSTICA}

Tal y como hemos visto en apartados anteriores, muchas han sido las iniciativas que tienen como finalidad medir la sostenibilidad de la actividad turística diseñando grupos de indicadores o modelos que responden a este objetivo. En el año 2001 se publicó en Inglaterra el documento "National Sustainable Tourism Indicators: Monitoring Progress Towards Sustainable Tourism" en el cual se recogen las tres acciones básicas necesarias para conseguir desarrollar un turismo sostenible: (a) Proteger y mejorar el entorno natural y los destinos turísticos; (b) Apoyar a las comunidades y la cultura locales; (c) Beneficiar las economías locales de los destinos. Un modelo de evaluación de la Idoneidad de Desarrollo Turístico (IDT) debe de proporcionar una metodología sistemática para organizar, combinar y medir indicadores significativos de modo que las autoridades responsables puedan extraer conclusiones acertadas sobre la calidad de los ecosistemas humano y natural del destino turístico (Ko, 2001). Se entiende por indicador de sostenibilidad a aquellas medidas cuantitativas o cualitativas que permiten conocer, cuando se aplican periódicamente, los cambios producidos a favor o en contra en relación con las condiciones ambientales, socioculturales y económicas de un destino turístico con el fin de tomar decisiones estratégicas de mejoramiento, mantenimiento o transformación de una situación dada (Sancho et al., 2007).

Los indicadores de sostenibilidad turística tienen como objetivo, mediante la simplificación de la realidad y centrándose en los aspectos más relevantes, ofrecer información sintética y mesurable sobre el comportamiento del turismo en un determinado

\footnotetext{
${ }^{1}$ Trabajo que se enmarca dentro del proyecto "Diagnóstico de la actividad turística en Viñales (Cuba) y tendencias de su desarrollo sostenible". A/023576/09. AECID-Centro de Cooperación URV Solidaria.
} 
territorio que permita establecer las condiciones del sistema actual y comprobar su evolución cuando éstos indicadores son aplicados periódicamente.

La selección del conjunto de indicadores para medir la sostenibilidad del turismo en el Valle de Viñales se llevó a cabo en función de varios criterios y en dos fases. En primer lugar se realizó un listado exhaustivo de indicadores teniendo en cuenta cuestiones tales como (a) su capacidad explicativa y nivel de significación del indicador para medir la sostenibilidad de la actividad turística; (b) su claridad a la hora de ser interpretados y (c) si éstos ofrecían la posibilidad de establecer comparaciones. En segundo lugar y a partir de ese primer listado, se realizó una selección de aquellos indicadores de los cuales se disponía de datos. Este aspecto es fundamental ya que, tal y como se puede comprobar en los resultados, una de las principales carencias detectadas en la zona es la falta de una recopilación sistemática de información. Es necesario remarcar que en este estudio se incluyen indicadores tanto cuantitativos como cualitativos que no son excluyentes si no complementarios. De este modo se puede intentar valorar una misma variable desde una perspectiva cuantitativa así como cualitativa.

La siguiente fase establece una escala de valoración para cada uno de los indicadores seleccionados. Así, una parte de ellos tiene la capacidad de medir la evolución que ha experimentado esta variable en un conjunto de años, son los indicadores dinámicos, y para ellos se toma la escala de valoración de: crecimiento respetuoso, cambio poco significativo, deterioro o riesgo, datos insuficientes o no comparables, Otros, los de estado, intentan medir la situación actual de una determinada problemática puesto que no existe la posibilidad de realizar una comparación en el tiempo. Este conjunto de indicadores utiliza la escala de valoración alto, medio, bajo.

Con el objetivo de, por un lado, homogenizar los datos referentes a cada uno de los indicadores y, por otro, ofrecer la información de una forma eficiente, se elaboró un formulario estándar que fue utilizado para construir cada uno de los indicadores. Este formulario o ficha está compuesto de tres grandes apartados (Figura 2). En el primero se realiza una descripción exhaustiva del indicador apuntando el nombre, su objetivo y descripción, la fuente, el método de elaboración adjuntando, si es el caso, la fórmula utilizada para su cálculo, y su dimensión o valoración de la sostenibilidad. En la segunda parte se realiza una descripción de los resultados obtenidos de cada uno de los indicadores incluyendo tablas, gráficos y mapas si la variable es territorializable. El formulario se completa con una lista de recomendaciones recogida en el último apartado.

La recopilación de bibliografía científica y cartográfica se realizó durante la fase inicial del proyecto mientras que el trabajo de campo se desarrolló a partir de tres estancias en el área de estudio. La primera tuvo lugar en julio de 2009, la segunda 
entre los meses de junio y agosto de 2010 y la tercera en diciembre del mismo año ${ }^{2}$. En estas estancias se visitaron diversos organismos y se realizaron diferentes entrevistas con responsables del Parque Nacional del Valle de Viñales, Consejo del Poder Popular, Responsable de la Oficina de Estadística, etc. Además de las entrevistas, también fue fundamental para este estudio la observación y la recopilación de información de servicios turísticos en las diferentes agencias, oficinas de viajes, empresas turísticas, etc. La observación del desarrollo de la actividad turística así como del comportamiento de los turistas y de la población local del municipio durante la estancia en el lugar, es fundamental para comprender la estructura y dinámica del turismo en el Valle de Viñales ${ }^{3}$.

Tal y como se ha indicado anteriormente, el sistema de indicadores seleccionado trata de medir la sostenibilidad del turismo centrándose en el análisis del modelo territorial y turístico existente en el valle, así como la presión derivada de las actividades antrópicas en general y del turismo en particular. El total de indicadores utilizados (Tabla 1) es de 25, distribuidos en 18 que valoran el modelo territorial y turístico y 7 que evalúan la presión medioambiental y social existente en el Valle de Viñales en una serie cronológica que se remonta a esta última década.

\footnotetext{
${ }^{2}$ En noviembre del año 2011 se volvió al área de estudio para la presentación de los resultados a las autoridades locales. Alguna de las evaluaciones ha sido depurada en función de las observaciones realizadas en esta última estancia.

${ }^{3}$ El trabajo de campo y la recopilación de información fueron realizados por investigadores de las Universidades de La Habana (Cuba) y Rovira i Virgili (Tarragona, España) y personal del Parque Nacional de Viñales a los que queremos agradecer su colaboración desinteresada.
} 
Figura 2. Ficha de Indicador.

INDICADOR (nombre del indicador)

Incluir fotografia representativa del indicador

\section{OBJETIVO Y DESCRIPCIÓN:}

Definir el objetivo y describir el indicador

FUENTE:

Indicar las fuentes utilizadas para el cálculo del indicador

\section{MÉTODO DE CÁLCULO:}

Indicar el método de cálculo del indicador

\section{DIMENSIÓN O VALORACIÓN DE LA SOSTENIBILIDAD:}

Valoración indicadores dinámicos:

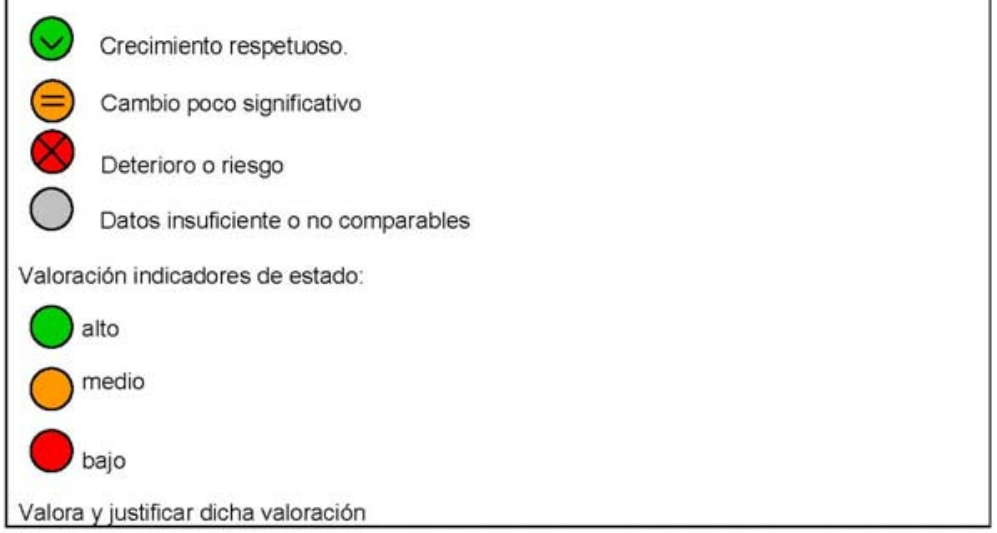

\section{DESCRIPCIÓN DE LOS RESULTADOS}

Describir los resultados incluyendo texto, gráficos, mapas, fotografias, etc.

\section{RECOMENDACIONES:}

Indicar, si fuese el caso, recomendaciones

Fuente: Elaboración propia. 
Tabla 1.Indicadores de Turismo Sostenible seleccionados para el Valle de Viñales (Cuba).

\begin{tabular}{|c|c|}
\hline INDICADOR & OBJETIVO \\
\hline \multicolumn{2}{|c|}{ MODELO TERRITORIAL-TURÍSTICO } \\
\hline \multicolumn{2}{|c|}{ Recursos turísticos actuales y potenciales } \\
\hline $\begin{array}{l}\text { Grado de diversificación de los recursos y } \\
\text { productos turísticos. }\end{array}$ & $\begin{array}{l}\text { Variedad de actividades turísticas ofrecidas en el destino turístico y } \\
\text { recursos con potencial para ser utilizados por el sector. }\end{array}$ \\
\hline Grados de protección de los recursos turísticos. & $\begin{array}{l}\text { Importancia que se da a la protección de los recursos turísticos } \\
\text { naturales, culturales y patrimoniales. }\end{array}$ \\
\hline \multicolumn{2}{|r|}{ Estructura territorial y económica } \\
\hline Accesibilidad interna y externa. & $\begin{array}{l}\text { Accesibilidad por carretera al valle de Viñales desde los núcleos de } \\
\text { población clave para la actividad turística. }\end{array}$ \\
\hline $\begin{array}{l}\text { Transporte público en el ámbito municipal, } \\
\text { provincial e interprovincial. }\end{array}$ & $\begin{array}{l}\text { Cuantifica la presencia de transporte público en el ámbito municipal, } \\
\text { provincial e interprovincial existente desde el núcleo urbano Viñales. }\end{array}$ \\
\hline Ingresos generados por la actividad turística. & $\begin{array}{l}\text { Ingresos que genera la actividad turística cuantificado a partir de los } \\
\text { ingresos percibidos por ésta. }\end{array}$ \\
\hline $\begin{array}{l}\text { Ingresos generados por la actividad turística: } \\
\text { recursos y equipamientos. }\end{array}$ & Efecto económico que generan los equipamientos y recursos turísticos. \\
\hline $\begin{array}{l}\text { Distribución población ocupada por sectores de } \\
\text { actividad. }\end{array}$ & $\begin{array}{l}\text { Peso relativo del turismo (sector terciario) a partir de la distribución y } \\
\text { evolución de la población ocupada por sectores productivos. }\end{array}$ \\
\hline \multicolumn{2}{|c|}{ Estructura demográfica } \\
\hline Crecimiento natural y saldo migratorio. & Diagnóstico demográfico. \\
\hline Población total censada según asentamientos. & Diagnóstico demográfico. \\
\hline Densidad de población. & Diagnóstico demográfico. \\
\hline Pirámide de población. & Diagnóstico demográfico. \\
\hline Índice de envejecimiento. & Diagnóstico demográfico. \\
\hline Indicador de dependencia. & Diagnóstico demográfico. \\
\hline \multicolumn{2}{|c|}{ Estructura de la oferta y la demanda turística } \\
\hline Oferta de alojamiento turístico reglado. & $\begin{array}{l}\text { Capacidad de la oferta de alojamiento turístico reglada según el tipo de } \\
\text { establecimiento y clasificación administrativa. }\end{array}$ \\
\hline Nivel de ocupación de alojamientos turísticos. & $\begin{array}{l}\text { Porcentaje de camas ocupadas que presentan los establecimientos de } \\
\text { alojamiento turístico en un período determinado. }\end{array}$ \\
\hline Empresas turísticas. & $\begin{array}{l}\text { Número y estructura de las empresas turísticas distintas del alojamien- } \\
\text { to. }\end{array}$ \\
\hline Distribución espacial de la oferta turística. & Concentración o dispersión de la oferta turística. \\
\hline Estructura de la demanda turística. & $\begin{array}{l}\text { Caracteriza la demanda turística según indicadores básicos: proceden- } \\
\text { cia, estancia media, gasto en destino, etc. }\end{array}$ \\
\hline \multicolumn{2}{|r|}{ PRESIÓN } \\
\hline \multicolumn{2}{|r|}{ Presión Humana } \\
\hline Distribución temporal de la presión humana. & $\begin{array}{l}\text { Distribución temporal de la presión humana (diaria, mensual o } \\
\text { trimestral y estacional). }\end{array}$ \\
\hline Distribución espacial de la presión humana. & $\begin{array}{l}\text { Mide el nivel de utilización y/o sobrecarga de recursos/instalaciones } \\
\text { por parte del visitante. }\end{array}$ \\
\hline Variación interanual de la presión humana. & Relación visitante/población residente. \\
\hline \multicolumn{2}{|c|}{$\begin{array}{ll}\text { Cambios en los usos del suelo } \\
\end{array}$} \\
\hline Cambio usos del suelo. & $\begin{array}{l}\text { Cambios en los usos del suelo expresados mediante cartografía } \\
\text { dinámica. }\end{array}$ \\
\hline \multicolumn{2}{|r|}{ Consumo de energía } \\
\hline $\begin{array}{l}\text { Consumo de energías renovables y no renova- } \\
\text { bles. }\end{array}$ & $\begin{array}{l}\text { Porcentaje de energías no renovables sobre el consumo total de } \\
\text { energía. }\end{array}$ \\
\hline \multicolumn{2}{|c|}{ Consumo de agua potable } \\
\hline Agua potable consumida. & Volumen y variación interanual del agua consumida total y per cápita. \\
\hline \multicolumn{2}{|r|}{ Generación de residuos } \\
\hline Generación de residuos y problemática asociada. & $\begin{array}{l}\text { Volumen y variación interanual de los residuos generados mensual o } \\
\text { trimestralmente según su tipo. }\end{array}$ \\
\hline
\end{tabular}

Fuente: Elaboración propia, a partir de Veras e Ivars (2003), y, Rivas y Magadán (2007): 


\section{APLICACIÓN DEL SISTEMA DE INDICADORES AL VALLE DE VIÑALES (CUBA): RESULTADOS, VALORACIÓN Y RECOMENDACIONES}

Siguiendo el modelo descrito, para cada indicador se valoró y/o dimensionó la sostenibilidad a partir de los resultados obtenidos. Como se recoge sintéticamente en la tabla 2 , de los 25 indicadores analizados, el 52\% fueron clasificados en la categoría de "cambio poco significativo" o en estado "medio" según fuese el indicador de dinámica o de estado de la situación. Solo dos indicadores, un $8 \%$ del total, obtuvieron una valoración "alta" o se consideró que su "crecimiento era respetuoso", mientras que el $40 \%$ restante apuntaban a un "crecimiento de deterioro o riesgo" o una situación de estado "baja".

Haciendo una evaluación de los resultados obtenidos por cada ámbito analizado, se puede constatar que en el municipio de Viñales, pese a tener unos recursos de alto valor y singularidad bajo protección y de gran potencial turístico y educativo, el diseño y diversificación de su producto turístico es limitado. En este sentido, es posible decir que se confunde la disponibilidad de patrimonio natural y cultural con la existencia de producto turístico. El producto turístico de Viñales que se comercializa desde la Habana y hacia el exterior (a través de páginas web oficiales) se basa principalmente en excursiones de 1 día de duración, sin pernoctación, que explotan la infraestructura turística extrahotelera más que los recursos turísticos ambientales o/y culturales. Tal y como indica Vázquez et alt. (2011) para que el patrimonio pueda ser considerado producto turístico es necesario, en primer lugar, que se ponga en valor o sea habilitado para su uso turístico $\mathrm{y}$, en segundo lugar, que se configure, junto con el resto de componentes del sistema turístico (alojamiento, servicios, capital humano...), en un producto turístico accesible e identificado por el mercado con un precio. Cuando esto no sucede, como en el caso de Viñales, el destino se encuentra desestructurado y se desarrollan productos simples que normalmente vinculan recursos de forma débil ya que se encuentran relacionados únicamente por su proximidad geográfica.

Uno de los aspectos positivos que hay que destacar es que la protección de los recursos turísticos es elevada. El alto valor y singularidad de los recursos naturales y culturales que posee Viñales le ha sido reconocido con diferentes declaraciones internacionales y nacionales de protección, aunque este hecho ha sido poco rentabilizado a nivel turístico y educativo. Esta situación queda patente analizando la promoción que del destino se hace puesto que, en la mayoría de los casos, en el material promocional no llega a mencionarse ninguno de estos reconocimientos.

El análisis de su estructura territorial y económica muestra en el ámbito de la accesibilidad pocos cambios, aunque la situación puede verse agravada por la perdida del número de líneas de transporte público y un deterioro progresivo de las infraestructuras.

En el ámbito económico se han detectado escasas modificaciones en la distribución de la población activa por sectores de actividad, mientras que en referencia al turismo se ha registrado una disminución de los ingresos generados por el conjunto de actividades turísticas del municipio y del peso de estos ingresos respecto al total. A 
partir de los datos disponibles se podría decir que no existe una dependencia de la economía de Viñales respecto a la actividad turística. Aunque con toda probabilidad el grado de dependencia se vería incrementado si se pudieran cuantificar los ingresos generados por el alquiler de habitaciones y paladares. Esta idea se ve reforzada ya que los ingresos económicos derivados de la explotación de recursos, como los senderos o equipamientos turísticos, apuntan hacia un crecimiento sostenido y respetuoso. Un riesgo palpable en este ámbito deriva de la coexistencia de dos economías. La de la población que renta habitaciones y regenta paladares, que perciben ingresos extras en dólares, y la del resto, muchos de ellos campesinos, garantes de un recurso turístico por excelencia de Viñales, su paisaje cultural, y que quedan fuera de los beneficios proporcionados por el turismo. Así, se percibe una desvinculación de la actividad turística por parte de la población campesina, sobre todo la de hábitat difuso, que es la que mantiene el paisaje de vega de tabaco.

La estructura demográfica presenta pocos cambios, aunque hay que remarcar el proceso de concentración de la población en los núcleos urbanos. La población urbana del municipio era, en el año 2009, de 14.778 personas, habiendo superado el 50\% de la población total $(53,3 \%)$ y es de prever que continuará aumentando; mientras que la población rural se sitúa en el $46,7 \%$ y probablemente seguirá disminuyendo. En principio la sostenibilidad general del municipio se verá poco afectada, ya que la disminución de la población dispersa y el aumento de la concentrada es en principio positivo para la sostenibilidad. Por otro lado la población absoluta del municipio y su densidad no son altas (27.707 habitantes y 33,5 habitantes por $\mathrm{km}^{2}$ ). Se detecta un avance del índice de envejecimiento del municipio lo que refleja un comportamiento de riesgo.

Los indicadores que analizan la estructura de la oferta y la demanda turística muestran una tendencia de riesgo, pues se constata un rápido crecimiento del número de casas que alquilan habitaciones a turistas; un nivel de ocupación de los alojamientos turísticos por debajo del $50 \%$ haciendo poco rentables las instalaciones y una alta concentración espacial de la oferta turística en sectores concretos del municipio. A pesar de ello se recoge el aumento reciente de la oferta de restauración como iniciativa de la población local y tras el permiso del estado para la creación de microempresas a finales del año 2010. En este sentido, tanto el alquiler de habitaciones en casas particulares como la actividad de los paladares o restaurantes, necesitan de un seguimiento para evitar que la falta de regulación, el peligro del exceso de oferta, etc. generen un efecto negativo en la actividad turística local ${ }^{4}$.

\footnotetext{
${ }^{4}$ En noviembre del año 2011, el número de licencias para alquilar habitaciones era de, aproximadamente, 300 mientras que las licencias de paladares, hacía apenas un año desde que se permitió su creación, era de unos 40 . El problema en este caso radica en que no se han realizado estudios de mercado que permitan dimensionar ambas actividades.
} 
Tabla 2.Valoración de Indicadores.

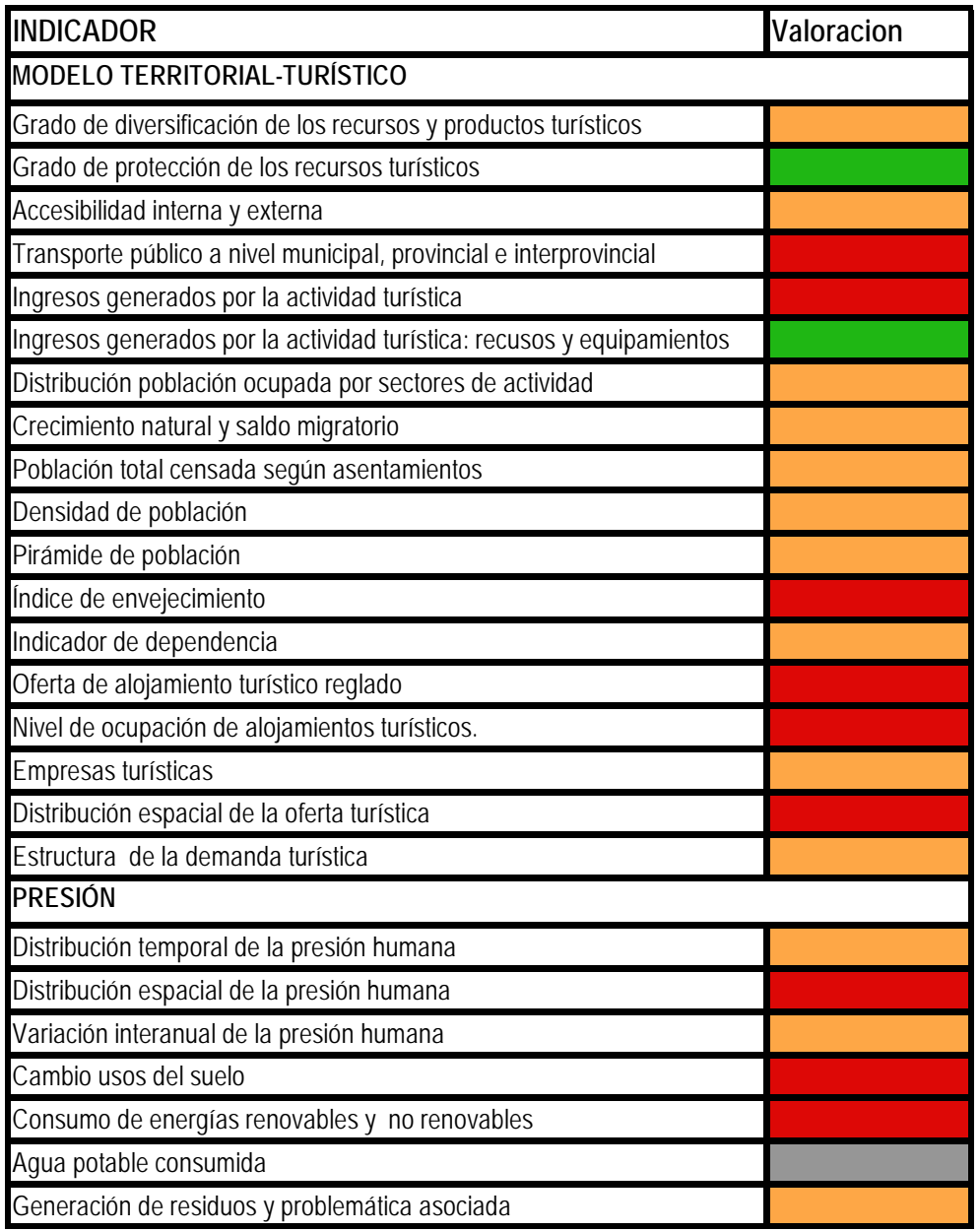

Fuente: Elaboración propia.

Del grupo de indicadores que miden y valoran la presión medioambiental y social se han detectado riesgos en: a) la distribución espacial de la presión humana, puesto que existe una concentración de visitantes en determinados equipamientos y recursos del municipio. Este hecho está ligado a la falta de puesta en valor del producto turístico y al modelo turístico implantado que se basa en excursiones con visitas a equipamientos más que a recursos turísticos, y b) una tendencia negativa en los usos del suelo debido a la expansión descontrolada de los asentamientos urbanos y a la reducción de la superficie dedicada a las vegas y a los equipamientos relacionados con esta actividad, como son los secaderos o casas de tabaco, elementos que conforman el 
paisaje cultural protegido. Esta tendencia supone una homogeneización de los usos del suelo que repercute en una uniformización del paisaje con una pérdida evidente de su calidad

A partir de las valoraciones y resultados obtenidos se han podido proponer una recomendaciones específicas para cada indicador y otras generales, cuyo principal objetivo es aportar directrices para orientar el desarrollo de la actividad turística de acorde a las condiciones específicas del territorio y a sus características económicas y sociales. Algunas de las recomendaciones generales propuestas son:

1) Establecer un sistema de coordinación entre las diferentes instituciones que gestionan el área declarada patrimonio de la humanidad y el parque nacional de Viñales, así como las diferentes administraciones, empresarios, asociaciones, es decir, todos los agentes implicados en la actividad turística.

2) Crear un Sistema de Información Territorial que permita contar con información exhaustiva y fiable a los diferentes organismos implicados en la gestión del territorio y de la actividad turística. (a) Completar el inventario de recursos naturales y culturales existentes para valorizarlos y/o darles la protección adecuada, así como realizar de forma periódica su seguimiento. (b) Recopilar información de forma sistemática y detallada sobre la afluencia, uso y demanda en las diferentes tipologías de alojamiento, recursos y servicios extrahoteleros. (c) Recopilar información económica, social, cultural y medioambiental que permitan poner en contexto el turismo y sus impactos en el Valle de Viñales.

3) Diversificar los productos y actividades turísticas así como la imagen del destino de Viñales basándose en el potencial del municipio como destino cultural y de naturaleza. Este nuevo enfoque permitiría integrar recursos y sistema turístico con la intención de incrementar el número de pernoctaciones y desestacionalizar la demanda de modo que Viñales deje de ser un destino de paso o complementario a La Habana.

4) Diseñar documentación de promoción turística del destino y de su oferta: web, mapa turístico, tríptico con información básica y específica (senderos, parque, equipamientos...), etc.

5) Creación y optimización de equipamientos informativos. (a) Ubicar una oficina del Centro de Visitantes del PNV en el núcleo urbano de Viñales e introducir actividades de interpretación y educación ambiental para informar y sensibilizar del alto valor de los recursos que acoge el área. (b) Creación de un centro de interpretación del sistema cavernario lo que pondría en valor las numerosas cuevas y restos arqueológicos presentes en la zona. (c) Complementar la información específica ofrecida por estas oficinas con información turística básica.

6) Incrementar la oferta hotelera reglada del municipio proponiendo la creación de un hotel en el núcleo del pueblo que complemente la oferta de hoteles situados en la periferia de la población.

7) Regular la oferta de empresas turísticas, de casas de alquiler y paladares con el fin de velar por la calidad de los servicios que ofrecen y dimensionar la oferta en relación a la demanda. 
8) Ejecutar medidas de protección para las vegas de tabaco así como para las infraestructuras relacionadas con esta actividad puesto que de ellas depende la declaración de Paisaje Cultural de la Humanidad (UNESCO).

9) Mejorar la gestión del uso de los senderos: (a) Integrar y/o coordinar la gestión de la oferta de senderos del PNV y de la Oficina de Patrimonio. (b) Controlar su uso para evitar problemas de sobreutilización que deterioren la calidad del entorno y de la experiencia turística así como revisar las capacidades de carga, prestando especial atención a las visitas realizadas a la Caverna de Santo Tomás puesto que es el sendero en el que se ha detectado mayor presión y riesgo de deterioro. (c) Planificar un servicio de transporte de turistas entre Viñales y el inicio de los senderos. Algunos de los recorridos existentes presentan el inconveniente de que, para llegar al punto de inicio, el visitante tiene que alquilar un vehículo lo que le comporta un sobre coste de la actividad.

10) Dar cumplimiento a la normativa urbanística que, entre otros aspectos, regula (a) la expansión urbana de modo que se respete tanto las necesidades de vivienda de la población como la preservación del entorno natural y paisajístico. (b) las nuevas construcciones y reformas en las viviendas tradicionales respetando la arquitectura local.

11) Mejorar los equipamientos básicos del municipio: (a) Optimizar la señalización vertical y horizontal de la red viaria existente, principalmente en la red local del municipio de Viñales. (b) Mantener en buen estado la red eléctrica del municipio y valorar la posibilidad de instalar paneles fotovoltaicos. (c) Subsanar las deficiencias técnicas del sistema de abastecimiento de agua potable para su mayor aprovechamiento y diseñar una política de concienciación ambiental sobre el ahorro de agua. (d) Extender el servicio de recogida de residuos a todo el municipio. Disponer contenedores para el depósito de residuos y facilitar su posterior recogida así como incrementar la separación doméstica de los residuos para conseguir un mayor porcentaje de reciclaje.

12) Diseñar y aplicar una política de concienciación y sensibilización ambiental incidiendo especialmente en los valores naturales y culturales del territorio.

13) Buscar e implantar fórmulas que eviten que los ingresos familiares derivados de la explotación de las casas de alquiler y paladares genere un impacto social negativo o que éste sea limitado a los implicados directamente en el negocio.

\section{CONCLUSIONES}

Como conclusión general es necesario decir que el turismo en Viñales debería de basarse en la puesta en valor de su territorio que cuenta con una identidad propia y singular avalada por los diferentes reconocimientos entre los que cabe destacar el Parque Nacional de Viñales y la categoría de Paisaje Cultural de la Humanidad de la UNESCO. Esta valoración de lo propio e identitario ha de respaldarse con una oferta extensiva y estructurada de alojamiento y actividades de ocio, no concentrada y de 
pequeña escala, que repercuta positivamente tanto en el incremento del nivel de vida de la población local como en la preservación de sus recursos naturales e identidad cultural.

En relación al método propuesto e implantado en este trabajo cabe mencionar algunas debilidades detectadas durante la fase de aplicación del sistema de indiciadores: a) Se ha encontrado una carencia de datos, sobre todo en el ámbito turístico, y, cuando éstos existe, se ha comprobado que su fiabilidad es escasa debido a una recopilación no sistemática, una variación en la estructura de los datos lo que lo que impide su comparación cronológica o un uso inapropiado de las fuentes y b) todos los indicadores son de igual importancia cosa que en la realidad no es así, Estos condicionantes hace que el resultado sea un modelo simplista de la realidad $(\mathrm{KO}, 2001)$. Se puede añadir que estos limitantes son inherentes a las ciencias sociales y que para entender un sistema complejo es necesario simplificarlo. Pese a estas limitaciones, el estudio realizado ha permitido avanzar hacia un mayor conocimiento a escala local del Valle de Viñales y ofrecer una herramienta para determinar las tendencias y dinámicas de la actividad turística, evaluar los cambios y transformaciones y, sobre todo, ofrecer unas recomendaciones generales y específicas de gran utilidad para los agentes responsables de la planificación y gestión del destino.

Por último, sí bien el método propuesto ha permitido constatar las posibilidades de análisis, este solo constituye un punto de partida para, en fases posteriores, ir ampliando y definiendo el sistema de indicadores en función de las particularidades del destino e incorporando otras dimensiones como la calidad, la comunidad, la gestión, etc. que ayuden a valorar y a medir de forma más precisa e integral el desarrollo de la actividad turística.

\section{BIBLIOGRAFÍA}

ARANDA TORRENTS, X. (2003): Un sistema de indicadores sostenibles aptos para un destino turístico: un fundamento metodológico, en: XII Simposio Internacional de Turismo y Ocio, ESADE, 3-4 abril de 2003, Barcelona.

BARBOSA JASSO, A.M. (2003): Cuatro casos de análisis comparativo de Destinos Turísticos de Playa en México, Escuela de Turismo, Universidad Autónoma de Sinaloa, Secretaría de Turismo del Gobierno de México, 19 pp. http://www.sectur.gob.mx/Congreso_de_Investigacion/ponencias/UAS.Adriana\% 20Barbosa.pdf

BLANCAS, F.J.; GONZÁLEZ, M.; PÉREZ, F. (2008): Indicadores sintéticos de turismo sostenible a nivel local, Congreso Nacional del Medio Ambiente. Cumbre del Desarrollo Sostenible, 1 - 5 de diciembre de 2008, (informe técnico, 27 pp.)

CABALlERO, R.; PÉREZ, V.; CAMARGO, I.; GONZÁLEZ, M; PÉREZ, F. GUERRERO, F. (2010): La gestión de la sostenibilidad del turismo de naturaleza en Cuba: el uso de indicadores sintéticos, XVIII Jornadas ASEPUMA - VI Encuentro Internacional, Anales de ASEPUMA, no 18: 802.

CHOI, H.C.; SIRAKAYA, E. (2006): Sustainability Indicators for Managing Community Tourism, Tourism Management, 27, 1274-1289. 
CITMA Y MINTUR (2003): Indicadores de Sostenibilidad para el turismo en Cuba, documento preliminar.

DIAZ, G.; NORMAN, A. (2004): Manual de procedimientos para entrenadores en Turismo Sustentable, ACS-AEC.

HERNÁNDEZ, P.L.; RAMÍREZ, J.F.(2005): Pinar de Río. Cuba pasaje a la naturaleza, Exclusivas Latinoamericanas ELA S.L., Madrid.

HOLDER, J.S. (1988): Pattern and Impact of Tourism on the Environment of the Caribbean, Tourism Management, Volume 9, Issue 2, 119-127

KO, .T.G. (2005): Development of a Tourism Sustainability Assessment Procedure: a Conceptual Approach, Tourism Management, 26, 431-445

KO, J.T.G. (2001): Assessing Progress of Tourism, Sustainability, Annals of Tourism Research, Elsevier Science Ltd, Vol. 28, No. 3, 817-820.

MELERO, N. (2005): Viñales, un sitio natural y cultural excepcional, en Viñales, un paisaje a proteger, UNESCO, CEU-H, DPPF, Cooperación Italiana, Proyecto Los Mogotes, CRIC, Università Calabria. 21-30.

NEL-LO ANDREU, M. y PÉREZ ALBERT, Y. (2007): La política turística en Panamá. Resultados y perspectivas, Cuadernos de Turismo, nº 20, (2007); 199221.

OMT (1998): Desarrollo turístico sostenible. Guía para administradores locales. OMT, Madrid, 207-214.

OMT (2005): Indicadores de desarrollo sostenible para los destinos turísticos. OMT, Madrid.

PÉREZ, V.E.; BLANCAS, F.J.; GONZÁLEZ, M.; GUERRERO, F.M.; LOZANO, M.; PÉREZ, F. y CABALLERO, R.E. (2009): Evaluación de la sostenibilidad del turismo rural mediante indicadores sintéticos, Revista de Investigación Operacional, Vol., 30, No.1, 40-51.

PRISKIN, J. (2001): Assessment of Natural Resources for Nature-Based Tourism: the Case of the Central Coast Region of Western Australia, Tourism Management, 22, 637-648.

RIGOL, I. (2005): Viñales ¿por qué un paisaje cultural?, Viñales, un paisaje a proteger, UNESCO, CEU-H, DPPF, Cooperación Italiana, Proyecto Los Mogotes, CRIC, Università Calabria. 31-38.

RIVAS, J.; MAGADÁN, M. (2007): Planificación turística y desarrollo sostenible, ed. Septem, Oviedo (España).

SAARINEN, J. (2006): Traditions of Sustainability in Tourism Studies, Annals of Tourism Research, Vol. 33, No. 4, 1121-1140.

SALINAS CHÁVEZ, E.; NAVARRO JURADO, E.; ECHARRI CHÁVEZ, M.; OSORIO, J.A. (2008): Metodologías para la evolución de la sustentabilidad territorial: el uso de indicadores en destinos turísticos de Cuba, en: Boletín de la Real Sociedad Geográfica, (144), 77-102.

SANCHO, A.; GARCÍA, G; ROZO, E. (2007): Comparativa de indicadores de sostenibilidad para destinos desarrollados, en desarrollo y con poblaciones vulnerables, Annals of Tourism Research en español, vol. 9, $\mathrm{n}^{\circ}$ 1, 207, 150-177. 
VÁZQUEZ VARELA, C.; MARTÍN GIL, F. (2011): Problemas de sostenibilidad del turismo rural en España, Anales de Geografía de la UCM, vol. 31, núm. 1 171194 doi: 10.5209/rev_AGUC.2011.v31.n1.8.

VERA REBOLLO, F.; IVARS BAIDAL, J.A. (2003): Sistema de Indicadores Aplicado a la Planificación y Gestión del Desarrollo Turístico Sostenible, VALDES, L. Y PÉREZ, J.M.: Experiencias Públicas y Privadas en el Desarrollo de un Modelo de Turismo Sostenible, Fundación Universidad de Oviedo, 105-129.

VERA REBOLLO, F.; IVARS BAIDAL, J.A. (2004): Indicadores de sostenibilidad para destinos turísticos maduros: balance y propuestas de aplicación http://portalsostenibilidad.upc.edu/archivos/fichas/informes/indicadoresdestinostur ismomaduro.pdf

WIESE, P. (2000): The Self-Destruct Theory of Tourism, Wise Coastal Practices for Sustainable Human Development Forum, Cancún-Mexico, $\mathrm{http}: / / \mathrm{www}$.csiwisepractices.org/?read=246\#sp 